\title{
Microbial ecology of the female lower genital tract during pregnancy*
}

\author{
JOHN DE LOUVOIS \\ M.Sc. \\ RosAlinde HuRLEY \\ M.D.
}

\author{
Valerie C. Stanley \\ Ph.D.
}

J. B. JONES $\dagger$
F.R.C.S.

\author{
J. E. B. FoulKes \\ F.R.C.S. \\ Queen Charlotte's Hospital for Women, and the Institute of Obstetrics and Gynaecology,
University of London
}

Summary

The posterior fornices of the vagina of 280 unselected pregnant women were sampled, using a sponge swab, squeezed out into Stuart's Transport Medium. Other specimens were also taken. Eleven primary media were inoculated, wet swabs were examined, and Gramstained smears were also made. Nineteen groups or species of microbes were identified.

Nearly $80 \%$ of women harbour pathogenic, or potentially pathogenic microbes; $52 \%$ harbour streptococci of various sorts. The incidence of the various groups, and species of microbes is tabulated. There is a statistically significant correlation between the presence of polymorphonuclear leucocytes in excess of one per high power field, and the presence of lesions of the cervix.

\section{Introduction}

The study reported here in preliminary form was undertaken to determine the range of organisms present in the female lower genital tract during pregnancy and to investigate the possible relationships between the presence of the flora and clinical signs and symptoms of vaginitis, vulvitis, cervicitis and other local morbidity. No attempt was made to seek viruses, T-strain mycoplasmas, or Chlamydia spp.; nor were exacting methods of anaerobiosis used.

\section{Materials and methods \\ Patients studied}

Two hundred and eighty unselected pregnant women were examined by one of two obstetricians who carried out a full internal examination. The clinician completed a computer questionnaire for each patient which, in addition to personal details, contained an assessment of the presence and degree

\footnotetext{
* Paper read at 'Research Work in Progress' Symposium, British Postgraduate Medical Federation, 19 February 1974.

+ Present address: The Hospital for Women, Soho Square. Correspondence: Dr R. Hurley, Queen Charlotte's Hospital for Women, Goldhawk Road, London W6 0XG.
}

of severity of vaginal discharge and pruritus, of vaginitis, vulvitis and cervicitis, together with observations of any other abnormalities. Past history of vulvovaginitis, venereal disease, and other relevant clinical conditions was noted.

\section{Microbiological specimens}

Each patient had charcoal swabs taken from the urethra, cervix and rectum for culture of Neisseria gonorrhoeae. These were plated in the clinic on to warm chocolate agar and gonococcal selective agar plates (Oxoid). Using a piece of sterile foam plastic sponge $3 \times 1 \times 1 \mathrm{~cm}$, held in sterile forceps, the posterior fornix was swabbed via a Cusco speculum; a dry swab was taken for a Gram-smear. After collection the sponge was placed in $2 \mathrm{ml}$ Stuart's Transport Medium for transfer to the laboratory where it was squeezed out into the medium and discarded. After thorough mixing the medium was used to inoculate eleven different culture media (Table 1) and to make wet films. Women found to be harbouring Candida albicans in an apparently healthy vagina were examined later during pregnancy, but otherwise, sequential studies were not undertaken.

\section{Microbiological methods}

The cultures were incubated as shown in Table 1. The Rogosa, crystal violet $(1 / 500,000)$, tellurite $(1 / 3000)$ and nalidixic acid $(1 / 25,000)$ media were included to aid the isolation of lactobacilli, streptococci, corynebacteria and Listeria monocytogenes respectively.

The mycoplasma medium (Oxoid) which had been prepared according to the manufacturer's instructions was poured into $5.0 \mathrm{~cm}$ diameter plastic Petri dishes to a depth of 4-5 mm. The plates were examined daily under the low-power objective of a microscope for the characteristic (fried egg) colonies of Mycoplasma. All the media were prepared and used within 3 days.

Following appropriate incubation the culture plates 
TABLE 1. Media used to culture the microflora of the lower genital tract of 280 unselected pregnant women

\begin{tabular}{lccc}
\hline \multicolumn{1}{c}{ Medium } & Atmosphere & $\begin{array}{c}\text { Incubation } \\
\text { time (hr) }\end{array}$ & $\begin{array}{c}\text { Incubation } \\
\text { temperature }\left({ }^{\circ} \mathrm{C}\right)\end{array}$ \\
\hline Blood agar & Aerobic & 48 & 37 \\
Blood agar & Anaerobic* & 48 & 37 \\
Heated blood agar & Carbon dioxide* & 48 & 37 \\
Crystal violet blood agar & Anaerobic* & 48 & 37 \\
Tellurite blood agar & Aerobic & 48 & 37 \\
Nalidixic acid blood agar & Aerobic & 96 & 37 \\
MacConkey agar & Aerobic & 48 & 37 \\
Rogosa agar (Difco) & Aerobic & 48 & 30 \\
Sabourauds agar & Aerobic & 48 & 30 \\
Mycoplasma agar & Trichomonas medium (Oxoid) & 192 & 37 \\
G.C. selective media (Oxoid) & Carbon dioxide in nitrogen & 96 & 30 \\
\hline
\end{tabular}

* Gaspak; ** candle jar.

were examined by two investigators (JdeL and VCS). Each different colonial type of organism was identified at least to genus according to the general descriptions laid down in Wilson and Miles (1964) and/or Cowan and Steel (1965). B-Haemolytic streptococci were grouped by the methods of Lancefield (1933) and Maxted (1948). Staphylococci were designated as Staphylococcus aureus or Staph . epidermidis according to the results of deoxyribonuclease (DNase) and/or coagulase tests. Micrococci were packet forming organisms which in cases of doubt were shown not to ferment glucose.

Because of the confusion which exists over the taxonomic position of organisms referred to as Haemophilus vaginalis, it was decided to include such organisms under the heading 'Gram-variable coccobacilli'.

Yeasts isolated from either Sabouraud's agar or the Trichomonas medium were identified according to the methods of Lodder and Kreger-van Rij (1952), Lodder (1970) and Merritt and Hurley (1972).

Organisms showing the typical fried egg colonial appearance of Mycoplasma were cut out of the agar and spread over a fresh plate of Mycoplasma medium. This procedure was repeated, if necessary, until the culture was pure. Colonies from pure cultures were cut out on a piece of agar measuring $5 \times$ $5 \mathrm{~mm}$ and inoculated into Mycoplasma broth containing $M$. hominis supplement (Oxoid). After $48 \mathrm{hr}$ incubation the broth was used neat and at dilutions of $1 / 50,1 / 100,1 / 200$, and $1 / 400$ to flood well dried plates of Mycoplasma agar. After the excess fluid had been removed the plates were dried and a taxodisc (BBL) containing $M$. hominis antiserum added to the centre of each plate. The plates were examined microscopically after $\mathbf{4 8} \mathrm{hr}$ incubation for zones of inhibition around the discs. A strain was identified as $M$. hominis if an inhibition zone of not less than $2 \mathrm{~mm}$ was obtained. All of the Mycoplasma subcultures were incubated at $37^{\circ} \mathrm{C}$ in an atmosphere of $10 \% \mathrm{CO}_{2}$ in nitrogen.
The dry high vaginal swab was used to prepare a smear which was stained by Gram's method and examined for the presence of bacteria, polymorphonuclear leucocytes, epithelial cells and yeasts. According to the morphology and Gram reaction of the bacteria seen, the films were assigned to one of three bacteriological grades (I-III) according to the criteria laid down by Gordon, Hughes and Barr (1966). Grade I: smears showing a morphological flora consisting exclusively or predominantly of organisms characteristic of lactobacilli; Grade II: smears which showed a mixed flora including lactobacilli; Grade III: smears which had a mixed morphological flora but which showed a total absence of lactobacilli.

The presence of polymorphs in the Gram film was recorded as follows, using a microsystem 70 with a $\times 8$ eyepiece and a $\times 100$ oil immersion objective. $\mathrm{Nil}=$ no polymorphs seen; scanty $=<1$ per high power field (HPF); $+=1-5$ per $\mathrm{HPF} ;++=$ 5-20 per HPF; gross $=$ frank pus. Epithelial cells were recorded in a similar way: nil or scanty $=<2$ per HPF; $+=>2$ per HPF (single cells); $++=$ sheets of cells.

\section{Results}

The figures in Table 2 represent total isolates, irrespective of the density of growth. Clostridium welchii, L. monocytogenes, $N$. gonorrhoeae and Haemophilus species, although sought, were not isolated.

Table 3 shows the incidence, and types, of streptococci isolated. Fifteen of twenty-five isolates of $\beta$ haemolytic streptococci belonged to Lancefield Group B. Group D streptococci are included among the faecal streptococci. Lancefield Group A streptococci were not isolated. Approximately a third of the $\beta$-haemolytic streptococci were not groupable by the Lancefield or Maxted techniques using A, B, C, D, $F$ or $\mathbf{G}$ antisera. No attempt was made further to identify anaerobic or non-haemolytic streptococci. 
TABLE 2. Flora of lower genital tract (HVS) in 280 unselected pregnant women, QCMH, 1973

\begin{tabular}{lcc}
\hline \multicolumn{1}{c}{ Microbe } & $\begin{array}{c}\text { No. of patients } \\
\text { from whom } \\
\text { isolated }\end{array}$ & $\begin{array}{c}\% \\
\text { Incidence }\end{array}$ \\
\hline Corynebacteria & 234 & $83 \cdot 4$ \\
Lactobacilli & 229 & $81 \cdot 8$ \\
Staphylococcus epidermidis & 185 & $66 \cdot 1$ \\
Micrococci & 103 & $36 \cdot 8$ \\
Faecal streptococci & 96 & $34 \cdot 3$ \\
Yeasts & 68 & $24 \cdot 3$ \\
Microaerophilic and & & \\
anaerobic streptococci & 61 & $21 \cdot 7$ \\
Escherichia coli & 54 & $19 \cdot 3$ \\
Mycoplasma hominis & 31 & $11 \cdot 0$ \\
B-Haemolytic streptococci & 25 & $8 \cdot 9$ \\
Gram-variable cocco-bacilli & 19 & $6 \cdot 8$ \\
Proteus spp. & 17 & $6 \cdot 1$ \\
Bacteroides spp. & 15 & $5 \cdot 4$ \\
Staph. aureus & 13 & $4 \cdot 6$ \\
Non-haemolytic streptococci & 12 & $4 \cdot 3$ \\
Trichomonas vaginalis & 7 & $2 \cdot 5$ \\
Neisseria spp. & 4 & $1 \cdot 4$ \\
Klebsiella aerogenes & 2 & $0 \cdot 7$ \\
Pseudomonas aeruginosa & 1 & $0 \cdot 4$ \\
\hline
\end{tabular}

TABLE 3. Flora of lower genital tract (HVS) in 280 unselected pregnant women, QCMH, 1973-Streptococci

\begin{tabular}{lcc}
\hline \multicolumn{1}{c}{ Type } & $\begin{array}{c}\text { No. of patients } \\
\text { from whom } \\
\text { isolated }\end{array}$ & $\begin{array}{c}\% \\
\text { Incidence }\end{array}$ \\
\hline Faecal & 96 & $34 \cdot 3$ \\
Microaerophilic or & 61 & $21 \cdot 7$ \\
anaerobic & 25 & 8.9 \\
B-Haemolytic & Nil & 5.4 \\
Group A & 15 & \\
Group B & 1 & \\
Group C & 1 & 2.9 \\
Groups F and G & 8 & $4 \cdot 3$ \\
Not groupable & 12 & \\
Non-haemclytic & & \\
\hline
\end{tabular}

TABLE 4. Flora of lower genital tract (HVS) in 280 unselected pregnant women, QCMH, 1973-Staphylococci

\begin{tabular}{lcc}
\hline \multicolumn{1}{c}{ Microbe } & $\begin{array}{c}\text { No. of patients } \\
\text { from whom } \\
\text { isolated }\end{array}$ & $\begin{array}{c}\% \\
\text { Incidence }\end{array}$ \\
\hline Staphylococcus epidermidis & 185 & $66 \cdot 1$ \\
Micrococci & 103 & $36 \cdot 8$ \\
Staphylococcus aureus & 13 & $4 \cdot 6$ \\
\hline
\end{tabular}

Table 4 shows the distribution of staphylococci. Staph. epidermidis and micrococci predominated, but there was a relatively high incidence of Staph. aureus in these patients.

Table 5 shows the distribution of Gram-negative rods. Of the seventy-three isolates of Enterobacteriaceae, in only twenty-five were the organisms present in moderate or heavy growth. Thus, in only $9 \%$ of patients were Enterobacteriaceae isolated in large numbers. Eight of these patients had Escherichia coli urinary tract infection. In the single patient from whom $P$. aeruginosa was isolated, the organisms were present only in small numbers.

TABLE 5. Flora of lower genital tract (HVS) in 280 unselected pregnant women, QCMH, 1973-Gram-negative rods

\begin{tabular}{lcc}
\hline \multicolumn{1}{c}{ Microbe } & $\begin{array}{c}\text { No. of patients } \\
\text { from whom } \\
\text { isolated }\end{array}$ & $\begin{array}{c}\% \\
\text { Incidence }\end{array}$ \\
\hline Escherichia coli & 54 & $19 \cdot 3$ \\
Proteus spp. & 17 & $6 \cdot 1$ \\
Bacteroides spp. & 15 & $5 \cdot 4$ \\
Klebsiella aerogenes & 2 & 0.7 \\
Pseudomonas aeruginosa & 1 & 0.4 \\
\hline
\end{tabular}

Some of the microbes that we isolated are considered to be associated with vaginitis during pregnancy, and their incidence is shown in Table 6. Of the sixty-eight patients harbouring yeasts seven grew organisms which we do not regard as pathogenic in this site. There were sixty-one isolates of pathogenic yeasts; seven of these were present only in small numbers. Candida albicans was isolated from $15 \%$ of patients who had an apparently healthy lower genital tract, and from $20 \%$ of patients with distinct morbidity. They were present in $41 \%$ of patients who complained of irritation, in only $18 \%$ of the remainder of the population. This difference is highly significant $(P<0.001)$.

TABLE 6. Flora of lower genital tract (HVS) in 280 un selected pregnant women, QCMH, 1973-microbes associated with vaginitis in pregnancy

\begin{tabular}{lcc}
\hline \multicolumn{1}{c}{ Microbe } & $\begin{array}{c}\text { No. of patients } \\
\text { from whom } \\
\text { isolated }\end{array}$ & $\begin{array}{c}\% \\
\text { Incidence }\end{array}$ \\
\hline Total yeasts & 68 & $24 \cdot 3$ \\
Pathogenic yeasts & 61 & $21 \cdot 8$ \\
Candida albicans & 49 & $17 \cdot 5$ \\
Torulopsis glabrata & 11 & $3 \cdot 9$ \\
C. krusei & 1 & $0 \cdot 4$ \\
Gram-variable cocco-bacilli & 19 & $6 \cdot 8$ \\
Trichomonas vaginalis & 7 & $2 \cdot 5$ \\
\hline
\end{tabular}

We established no other correlation between the presence of pathogenic yeasts and lower genital tract morbidity.

Because of the taxonomical confusion surrounding the organism variously named $\boldsymbol{H}$. vaginalis or Corynebacterium vaginale, we were unable to distinguish this microbe. We differentiated a group of Gram-variable coccobacilli, which included small, aerobic or microaerophilic rods, not belonging to any of the common genera. These organisms were essentially Gram-negative but with Gram-positive tendencies. None of them was dependent on $\mathrm{X}$ or $\mathrm{V}$ 
factors for growth. A number of isolates of Corynebacterium also had characteristics similar to those previously attributed to $H$. vaginalis.

We isolated a large number of pathogenic or potentially pathogenic bacteria from the female lower genital tract. Table 7 shows the percentage of patients harbouring these microbes. A large number of our patients were colonized by one or more of these strains, but Enterobacteriaceae were present only in small numbers. It is pertinent here to record that in only four of the 280 patients were the cultures sterile, and that cultures from thirty patients yielded a pure growth of lactobacilli.

Table 8 shows the distribution of the microflora according to the trimester of pregnancy. Apart from the trend towards increase in incidence of $M$. hominis and the anaerobic streptococci in the last trimester, isolation rates did not vary markedly with the stage of gestation reached.

We compared the isolation rates of different organisms with the appearance of the Gram-stained film, using the grading system previously outlined (Table 9). The distribution of some organisms, for example, Lactobacillus spp. and Candida albicans, bears no relation to the Gram-stained film. However, there is a significant increase $(P<0.001)$ in isolation rates of $M$. hominis, Bacteroides, anaerobic streptococci, Gram-variable coccobacilli and Trichomonas in specimens with a Grade III Gram-stained appearance.

TABle 7. Flora of lower genital tract (HVS) in 280 unselected pregnant women, QCMH, 1973

\begin{tabular}{|c|c|c|}
\hline $\begin{array}{l}\text { Women harbouring pathogens or potential } \\
\text { pathogens }\end{array}$ & $=$ & $77.9 \%$ \\
\hline Women harbouring streptococci & $=$ & $58.2 \%$ \\
\hline $\begin{array}{l}\text { Women harbouring vaginitis-associated } \\
\text { pathogens (i.e. Candida albicans, Torulop- } \\
\text { sis glabrata, C. krusei, Trichomonas }\end{array}$ & & \\
\hline vaginalis) & $=$ & $23 \cdot 2 \%$ \\
\hline Women harbouring staphylococci & $=$ & $68.6 \%$ \\
\hline Women harbouring Gram-negative ro & $=$ & $23.6 \%$ \\
\hline
\end{tabular}

Finally, we analysed the relationship between microbial flora and the presence of polymorphonuclear leucocytes in the Gram-film. Seventy-seven patients $(28 \%$ of the population) showed a polymorphonuclear count in excess of one per high power oil immersion field (Table 10). The presence of polymorphonuclear leucocytes did not relate to the

TABLE 8. Incidence of common micro-organisms

\begin{tabular}{lccc}
\hline & 1st trimester & 2nd trimester & 3rd trimester \\
\hline No. of patients & $112(40 \%)$ & $153(55 \%)$ & $15(5 \%)$ \\
Lactobacilli & $92(82 \%)$ & $116(76 \%)$ & $11(73 \%)$ \\
Corynebacteria & $65(58 \%)$ & $89(58 \%)$ & $8(53 \%)$ \\
Micrococci & $31(28 \%)$ & $37(24 \%)$ & $3(20 \%)$ \\
Staphylococcus epidermidis & $28(25 \%)$ & $56(37 \%)$ & $6(40 \%)$ \\
Faecal streptococci & $32(29 \%)$ & $32(21 \%)$ & $3(20 \%)$ \\
Escherichia coli & $10(9 \%)$ & $10(7 \%)$ & $2(14 \%)$ \\
Mycoplasma hominis & $8(7 \%)$ & $19(12 \%)$ & $4(27 \%)$ \\
Staph. aureus & $3(3 \%)$ & $3(2 \%)$ & $1(7 \%)$ \\
Bacteroides spp. & $5(5 \%)$ & $9(6 \%)$ & $1(7 \%)$ \\
Anaerobic and microaerophilic streptococci & $16(14 \%)$ & $33(22 \%)$ & $5(33 \%)$ \\
Gram-variable cocci-bacilli & $5(5 \%)$ & $12(8 \%)$ & $1(7 \%)$ \\
B haemolytic streptococci & $12(11 \%)$ & $11(7 \%)$ & $2(13 \%)$ \\
Trichomonas vaginalis & $4(4 \%)$ & $3(2 \%)$ & 0 \\
Torulopsis glabrata & $5(5 \%)$ & $4(3 \%)$ & $1(7 \%)$ \\
Candida albicans & $16(14 \%)$ & $27(18 \%)$ & 0 \\
\hline
\end{tabular}

TABLE 9. Relationship between bacterial grade (Gram-film) and selected micro-organisms

\begin{tabular}{lccc}
\hline & Grade I & Grade II & Grade III \\
\hline No. of patients & 205 & 37 & 38 \\
Lactobacilli & $177(86 \%)$ & $16(43 \%)$ & $26(68 \%)$ \\
Escherichia coli & $14(7 \%)$ & $5(14 \%)$ & $3(8 \%)$ \\
Mycoplasma hominis & $7(3 \%)$ & $4(11 \%)$ & $20(53 \%)$ \\
Staphylococcus aureus & $6(3 \%)$ & $1(3 \%)$ & 0 \\
Bacteroides spp. & $5(2 \%)$ & $2(5 \%)$ & $8(21 \%)$ \\
Anaerobic and microaerophilic streptococci & $20(10 \%)$ & $13(35 \%)$ & $21(55 \%)$ \\
Gram-variable cocco-bacilli & $6(3 \%)$ & $3(8 \%)$ & $9(24 \%)$ \\
B haemolytic streptococci & $18(9 \%)$ & $4(11 \%)$ & $3(8 \%)$ \\
Trichomonas vaginalis & $1(<1 \%)$ & $2(5 \%)$ & $4(10 \%)$ \\
Torulopsis glabrata & $8(4 \%)$ & $2(5 \%)$ & 0 \\
Candida albicans* & $30(15 \%)$ & $6(16 \%)$ & $7(18 \%)$ \\
\hline
\end{tabular}

* Includes two yeast mixtures. 
TABLE 10. Relationship between microflora and excess polymorphs (Gram-film) in 280 unselected pregnant women

\begin{tabular}{lccc}
\hline & \multicolumn{3}{c}{ No. of polymorphs per high power field } \\
\cline { 2 - 4 } & Less than 1 & $1-5$ & More than 5 \\
\hline No. of patients & 203 & 47 & 30 \\
Lactobacilli & $155(76 \%)$ & $39(83 \%)$ & $25(83 \%)$ \\
Corynebacteria & $122(60 \%)$ & $23(49 \%)$ & $17(57 \%)$ \\
Micrococci & $51(25 \%)$ & $14(30 \%)$ & $6(20 \%)$ \\
Staphylococcus epidermidis & $73(35 \%)$ & $9(19 \%)$ & $8(27 \%)$ \\
Faecal streptococci & $51(25 \%)$ & $9(19 \%)$ & $6(20 \%)$ \\
Escherichia coli & $17(8 \%)$ & $3(6 \%)$ & $2(7 \%)$ \\
Mycoplasma hominis & $23(11 \%)$ & $7(15 \%)$ & $1(3 \%)$ \\
Staph. aureus & $4(2 \%)$ & $3(6 \%)$ & 0 \\
Bacteroides spp. & $12(6 \%)$ & $3(6 \%)$ & 0 \\
Anaerobic and microaerophilic streptococci & $42(21 \%)$ & $8(17 \%)$ & $3(10 \%)$ \\
Gram-variable cocco-bacilli & $15(7 \%)$ & $2(3 \%)$ & $1(3 \%)$ \\
B haemolytic streptococci & $16(8 \%)$ & $8(17 \%)$ & $1(3 \%)$ \\
Trichomonas vaginalis & $2(1 \%)$ & $4(9 \%)$ & $1(3 \%)$ \\
Torulopsis glabrata & $9(4 \%)$ & $1(2 \%)$ & $1(3 \%)$ \\
Candida albicans* & $37(18 \%)$ & $9(19 \%)$ & $3(10 \%)$ \\
\hline
\end{tabular}

* Includes two yeast mixtures.

bacteria isolated, but of the seventy-seven patients with more than one polymorphonuclear leucocyte per high power field, forty-seven $(61 \%)$ had cervicitis or cervical erosion. Thirty-six per cent of all 130 patients with cervicitis or cervical erosion had excess leucocytes $(P<0.01>0.001)$.

\section{Summary of findings}

This study has shown that there is a surprisingly wide range of organisms present in the posterior fornix of pregnant women. Corynebacterium spp. are isolated as commonly as Lactobacillus spp. and these and Staph. epidermidis predominate.

Nearly $80 \%$ of women harbour pathogenic, or potentially pathogenic microbes; $52 \cdot 2 \%$ harbour streptococci of various sorts. Gram-negative rods are harboured by $23.6 \%$, but few of the Enterobacteriaceae isolated are present in large numbers. Organisms associated with vaginitis are harboured by $23 \cdot 2 \%$.

The microbial flora seems little different in the first, second or third trimester of pregnancy, apart from a trend towards increase in isolation rates of $M$. hominis and anaerobic streptococci in the third trimester.

There is no relationship between the graded appearance of the Gram-stained film, and the types of microbes isolated, save that $M$. hominis, Bacteroides, anaerobic streptococci, Gram-variable coccobacilli, and Trichomonas are more frequently isolatep where lactobacilli are adjudged to be absent, from the appearance of the Gram-film.
There is a statistically significant correlation between the presence of polymorphonuclear leucocytes in excess of one per high power field, and the presence of lesions of the cervix.

\section{Acknowledgments}

We are grateful to the nursing staff of Queen Charlotte's Maternity Hospital, and to Dr G. S. King and Dr D. Johnstone who programmed the data for computer analysis; to Barbara G. S. Leask who identified the yeasts; and to Dr Mair Thomas and Dr B. E. Andrews for their advice on isolation and identification of mycoplasmas.

\section{References}

CowAN, S.T. \& STEel, K.J. (1965) Manual for the Identification of Medical Bacteria. Cambridge University Press: London.

Gordon, A.M., Hughes, H.E. \& BARR, G.T.D. (1966) Bacterial flora in abnormalities of the female genital tract. Journal of Clinical Pathology, 19, 429.

LANCEFIELD, REBECCA C. (1933) A serological differentiation of human and other groups of haemolytic streptococci. Journal of Experimental Medicine, 57, 571.

LODDER, J. (Editor) (1970) The Yeasts: A Taxonomic Study. Second edition. North Holland Publishing Company: Amsterdam.

LODDER, J. \& KREgER-VAN RIJ, N.J.W. (1952) The Yeasts: A Taxonomic Study. North Holland Publishing Company: Amsterdam.

MAXTED, W.R. (1948) Preparation of streptococcal extracts for Lancefield grouping. Lancet, ii, 255.

Merritt, A.E. \& Hurley, Rosalinde (1972) Evaluation of sporulation media for yeasts obtained from pathological material. Journal of Medical Microbiology, 5, 21.

Wilson, G.S. \& Miles, A.A. (1964) Topley and Wilson's Principles of Bacteriology and Immunity. Edward Arnold: London. 